\title{
Cyclosporine A inhibits breast cancer cell growth by downregulating the expression of pyruvate kinase subtype M2
}

\author{
KAI JIANG ${ }^{1,2^{*}}$, BAOMING HE ${ }^{3 *}$, LIHUA LAI ${ }^{1}$, QINGYUN CHEN ${ }^{1}$, YANG LIU ${ }^{1}$, \\ QINGMING GUO ${ }^{1}$ and QINGQING WANG ${ }^{1}$ \\ ${ }^{1}$ Institute of Immunology, Zhejiang University School of Medicine, Hangzhou $310058 ;{ }^{2}$ Zhejiang Provincial Tongde Hospital, \\ Hangzhou 310012; ${ }^{3}$ Department of Nuclear Medicine, 309 Hospital of PLA, Beijing 100091, P.R. China
}

Received February 2, 2012; Accepted April 9, 2012

DOI: $10.3892 / \mathrm{ijmm} .2012 .989$

\begin{abstract}
The high proliferative rate of tumor cells leads to metabolic needs distinct from those of their normal counterparts. An embryonic- and tumor-specific isoform of the enzyme pyruvate kinase M2 (PKM2) is overexpressed in cancer cells to increase the use of glycolytic intermediates for macromolecular biosynthesis and tumor growth. We report that Cyclosporin A (CsA) can regulate the expression and activity of PKM2 in breast cancer cell lines MCF-7, MDA-MB-435 and MDA-MB-231. PKM2 was found to be highly expressed in the three breast cancer cell lines compared to normal primary breast cells. Treatment with CsA inhibited the viability of breast cancer cells in a time- and dose-dependent manner. CsA significantly downregulated the expression of PKM2 in breast cancer cells and decreased adenosine triphosphate (ATP) synthesis, which induced cancer cells to undergo necrosis. Furthermore, the growth suppression effect of CsA was impaired in MCF-7 cells when they were transfected with the PKM2 overexpression plasmid, suggesting that CsA was an effective inhibitor of PKM2-dependent proliferation of breast cancer cells. These results may provide new insights into the mechanism of CsA in cancer therapy.
\end{abstract}

\section{Introduction}

Tumorigenesis is characterized by enhanced activities of glycolytic enzyme as well as distinct changes in the glycolytic isoenzyme. Tumor cells must occupy a high rate of nutrients and maintain a balance between the use of nutrients for energy production and for anabolic processes, whereas less nutrient uptake is required in most adult tissues and a greater fraction of

Correspondence to: Professor Qingqing Wang, Institute of Immunology, Zhejiang University School of Medicine, Hangzhou 310058, P.R. China

E-mail: wqq@zju.edu.cn

*Contributed equally

Key words: cyclosporine A, pyruvate kinase M2, adenosine triphosphate, breast cancer cells available nutrients are used for energy production rather than macromolecule synthesis. This difference between metabolism of tumor cells and their normal counterparts was first pointed out by Warburg (1). Glucose is used for anabolic processes preferentially rather than oxidative phosphorylation in tumor cells, and this metabolic switch may be required to support cell growth. The pyruvate kinase isoenzyme M2 (PKM2) is one of the most important regulators of the balance between glycolytic energy regeneration [e.g. adenosine triphosphate (ATP) synthesis] and the synthesis of cell building blocks (e.g. protein, lipid and nucleic acid synthesis) in tumor cells. There are four pyruvate kinase isoenzymes (M1, M2, L and R) that are known. PKM2 is the embryonic form which is progressively replaced by PKM1 in brain and muscle, PKL in kidney and liver, and PKR in erythrocytes during differentiation. When adult tissue cells in quiescence re-enter the cell cycle, the tissue-specific isoenzymes are replaced by PKM2 (2). In most, if not all, tumor cells PKM2 is overexpressed. PKM2 expression in cancer cells results in aerobic glycolysis and is suggested to bestow a selective growth advantage, and thus, a promising target. Many oncogenes impart a common alteration in cell metabolism, inhibition of the M2 isoform might be of broad applicability (3-5). Knockdown of PKM2 and replacement of PKM2 by PKM1 were shown to reduce the carcinogenicity of human tumor cell lines to form tumors in nude mouse xenografts $(6,7)$. Microarray studies have shown that pyruvate kinase which regulates the rate-limiting final step of glycolysis, is one of the most upregulated gene sets in cancer (8).

Cyclosporin A (CsA) is a noncytotoxic immunosuppressant that was first discovered in 1970s and it was initially used for immunosuppression following organ and marrow transplantation. Subsequently, it has been applied in virtually all branches of medicine where autoimmune or inflammatory processes play a role in the pathology. Recent studies have explained that CsA is also associated with cancer therapy. CsA is a substrate and inhibitor of P-glycoprotein (P-gp) (9). It has been used as one of the first-generation multidrug resistance (MDR) modulators to reverse MDR and improve chemotherapy (10). Chemotherapy combined with CsA increases the plasma concentration of chemotherapy drugs and decreases the clearance of P-gp substrates, such as digoxin and etoposide $(11,12)$. On the other hand, CsA is an inhibitor, but not a substrate for breast cancer resistance protein (BCRP) (13). In the present 
study, we found that CsA inhibits breast cancer cell proliferation by influencing the glycolysis through downregulating the pyruvate kinase subtype M2 (PKM2).

\section{Materials and methods}

Cell culture and transfection. Three human breast carcinoma cell lines MCF-7, MDA-MB-435 and MDA-MB-231 were obtained from ATCC and cultured in RPMI-1640 medium supplemented with $10 \%$ FBS. Normal breast primary cells were obtained from normal breast tissue biopsies from the Second Affiliated Hospital (Zhejiang University School of Medicine, China) after patients had given their informed consent and were processed as described before (14). Tissues were mechanically dissociated with scissors and incubated at $37^{\circ} \mathrm{C}$ with constant shaking in HBSS containing $500 \mathrm{IU} / \mathrm{ml}$ collagenase. Digestion was monitored under an inverted microscope. Suspension was collected and centrifuged, cells were then plated in DMEM containing $10 \mu \mathrm{g} / \mathrm{ml}$ insulin, $5 \times 10^{-6} \mathrm{M}$ cortisol $50 \mathrm{IU} / \mathrm{ml}$ penicillin, $50 \mu \mathrm{g} / \mathrm{ml}$ streptomycin, $2 \mathrm{ng} / \mathrm{ml}$ EGF and $10 \%$ FBS. Cells were cultured in a 5\% $\mathrm{CO}_{2} 95 \%$ air humidified incubator. Medium was changed every 2 days. Human PKM2 encoding gene was amplified by RT-PCR from total-RNA extracted from MCF-7 cells and was ligated to pEGFP-N1 (Clontech Laboratories, USA) plasmid by T4 DNA ligase after digestion by the restricted endonucleases, EcoRI and BamHI. MCF-7 cells were transfected with pEGFP-N1-PKM2 expression vector using Lipofectamine 2000 (Invitrogen Life Tecnologies, USA) according to manufacturer's instructions for $24 \mathrm{~h}$ before CsA treatment.

Assay of cell growth. Cell growth was measured using 3-(4, 5-dimethylthiazol-2-yl)-2, 5-diphenyltetrazolium bromide (MTT) conversion assay. Cells were seeded in a 96-multiwell plate at a concentration of $5 \times 10^{3}$ cells/well. After overnight incubation, the medium was replaced with a fresh medium containing CsA (Merck KGaA, Germany) at various concentrations and the plates were incubated for $48 \mathrm{~h}$. MTT $(0.5 \mathrm{mg} / \mathrm{ml})$ was added during the last $4 \mathrm{~h}$ of incubation and absorbance was measured at $570 \mathrm{~nm}$.

Cell cycle analysis. MCF-7 cells were incubated with CsA at various concentrations for $24 \mathrm{~h}$. For cell cycle analysis, cells were harvested, rinsed with PBS and fixed in $70 \%$ ethanol overnight at $4^{\circ} \mathrm{C}$. The fixed cells were centrifuged and resuspended in $500 \mu \mathrm{l}$ of PBS containing $0.1 \mathrm{mg} / \mathrm{ml}$ RNase-A and then incubated at $37^{\circ} \mathrm{C}$ for $30 \mathrm{~min}$. Cells were then washed with PBS and resuspended in PBS containing $0.05 \mathrm{mg} / \mathrm{ml}$ propidium iodide at room temperature for $30 \mathrm{~min}$. DNA content was determined by LSR flow cytometry (BD Biosciences, USA).

Mitochondrial membrane potential $(\Delta \Psi m)$ assessment. $\Delta \Psi \mathrm{m}$ was measured as the fluorescence of JC-1, a lipophilic and cationic dye, accumulated in the mitochondria in a potentialdependent manner. Cells were harvested and incubated for 20 min at $37^{\circ} \mathrm{C}$ before FACS analysis.

Western blot analysis. Cells were extracted in lysis buffer (Cell Signaling Technology, Inc., USA) and the lysates were separated on a $12.5 \%$ SDS-PAGE. Proteins were then transferred to a
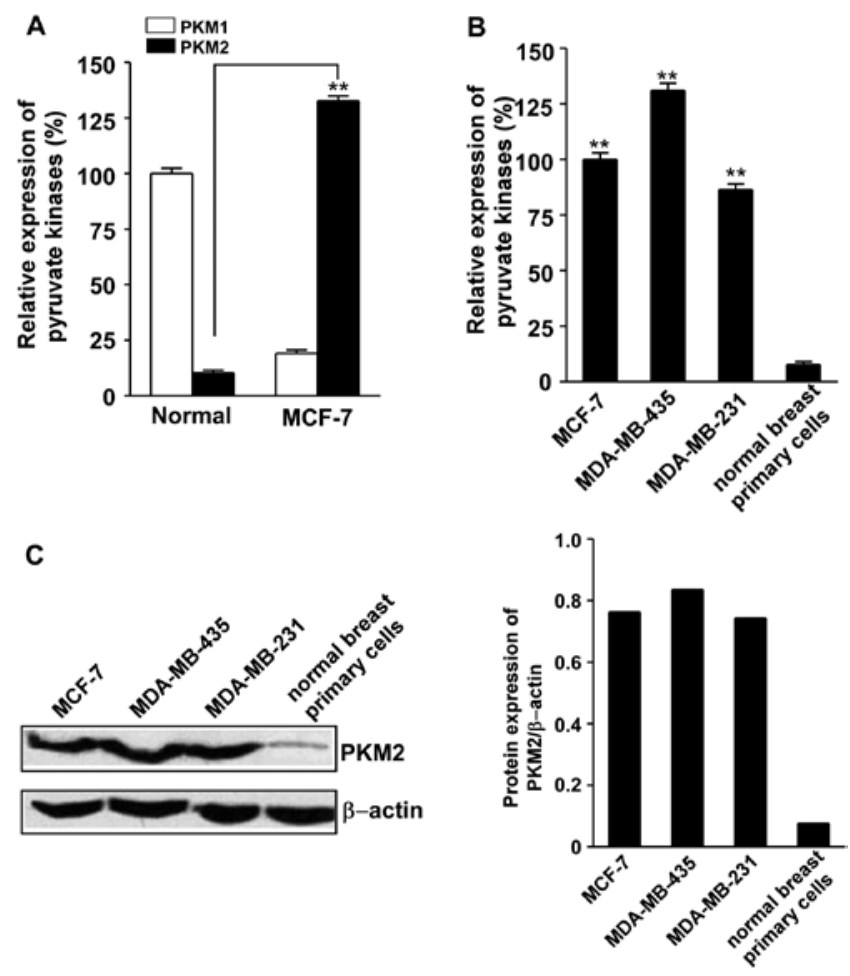

Figure 1. Overexpression of PKM2 in cancer cells compared to normal tissues. (A) mRNA expression of PKM1 and PKM2 was detected by real-time PCR in normal primary breast cells and MCF-7 cells. Data are the mean \pm SD $(n=4)$ of 1 representative experiment. ${ }^{* *} \mathrm{P}<0.01$ vs. normal cells. (B) The expression of PKM2 mRNA was determined by real-time PCR in MCF-7, MDA-MB-435, MDA-MB-231 breast cancer cells. Data are the mean \pm SD $(n=4)$ of 1 representative experiment. Similar results were obtained in 3 independent experiments. ${ }^{* *} \mathrm{P}<0.01$ vs. normal cells. (C) The protein level of PKM2 in MCF-7, MDA-MB-435, MDA-MB-231 and normal primary breast cells was detected by western blotting. The data shown are representative of 3 independent experiments.

PVDF membrane (Millipore, USA) in transfer buffer and were detected as we have previously described (15). Immunodetection was accomplished using the following primary antibodies: anti-PKM2 (clone \#3198; Cell Signaling Technology, Inc.) and anti- $\beta$-actin (Santa Cruz Biotechnology, Inc., USA).

Quantitative RT-PCR. Total-RNA was isolated using TRIzol reagent (Invitrogen Life Technologies). cDNA was synthesized using ReverTra Ace qPCR RT kit (Toyobo, Co., Ltd., Japan) according to manufacturer's instructions. qRT-PCR reactions were run on an Applied Biosystems 7500 Real Time PCR System machine as we have previously described (16). Gene expression levels in all samples were examined using SYBR-Green (Takara Bio, Inc., Japan) according to the manufacturer's protocols. The following primer pairs were used for qRT-PCR, which were designed according to a previous study (17): human PKM1 F, 5'-GTGCGAGCCTCAAGTCACT-3' and R, 5'-GCTGCTAAACACTTATAAGAAG-3'; human PKM2: F, 5'-GCCTGGCGCCCATTACCA-3' and R, 5'-CCCACTGCAGCACTTGAAG-3'; human GAPDH F, 5'-TGCCAAATATGATGACATCAAGAA-3' and R, 5'-GGA GTGGGTGTCGCTGTTG-3'. After normalization of the expression of mRNA by GAPDH, the relative levels of transcripts in CsA vs. untreated cells were calculated using the $2-\Delta \Delta \mathrm{Ct}$ method (18). 
A
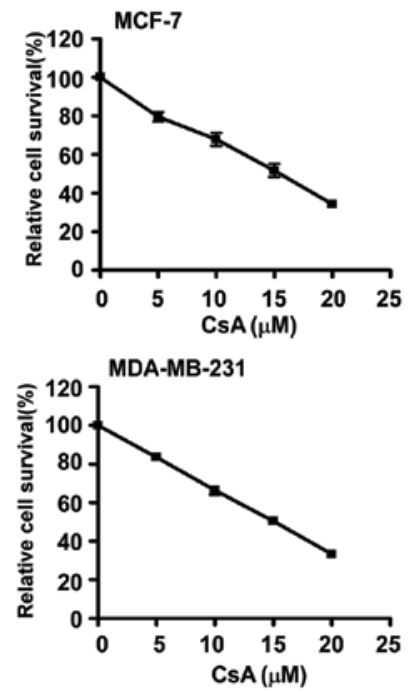
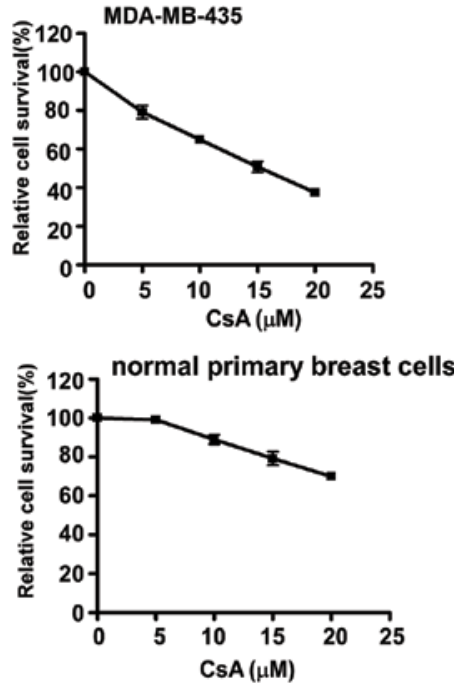

B
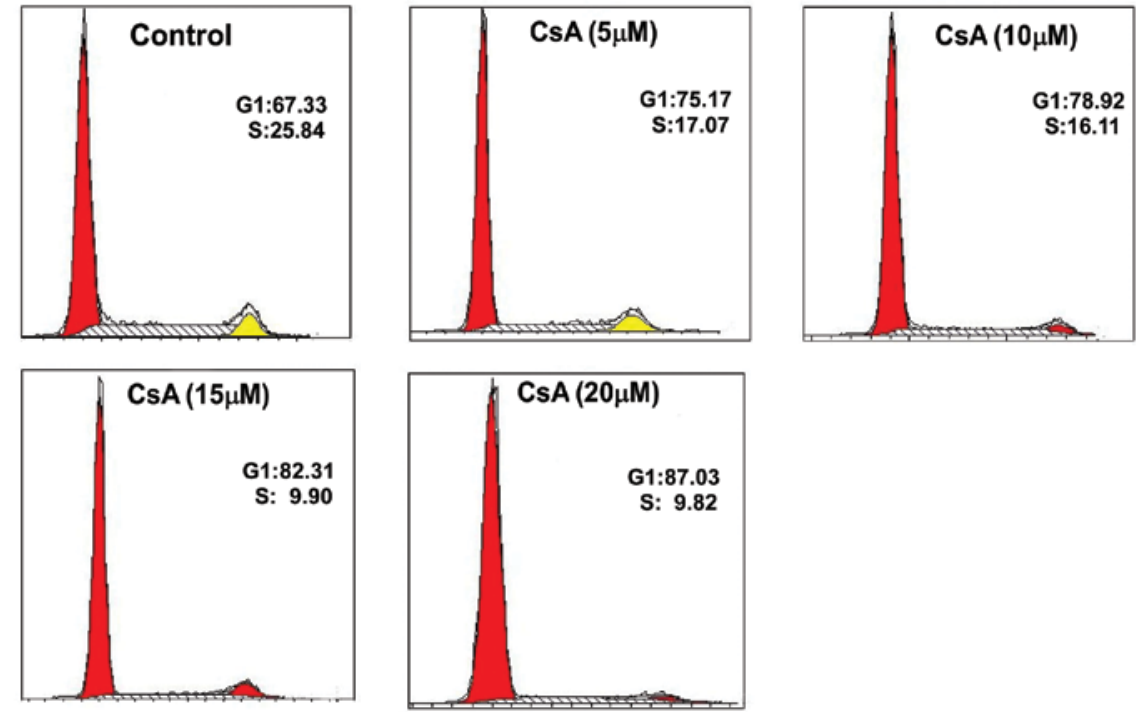

Figure 2. CsA inhibited the growth of breast cancer cells. (A) MTT assay of cell growth of MCF-7, MDA-MB-435, MDA-MB-231 and normal breast primary cells treated with various concentrations of CsA. Data are the mean $\pm \mathrm{SD}(\mathrm{n}=3)$ of a representative experiment. Similar results were obtained in 3 independent experiments. (B) Cell cycle analysis of MCF-7 cells treated with CsA for $24 \mathrm{~h}$ by propidium iodide staining. The percentages of cells in the G1 and S phases, respectively, are indicated. The data shown are representative of 3 independent experiments.

ATP measurement. ATP concentration was measured by the luminometric ATP assay and normalized as described (19). Cells were lysed in 1\% NP-40, and when the ATP in the lysates and luciferin/luciferase enzyme complex combined a reaction, which produced light, occured. The relative light units were detected by Multimode Detector (DTX 880; Beckman Coulter, USA). Intracellular ATP concentration was expressed as the percentage of untreated to controls.

Measurement of pyruvate kinase activity. Pyruvate kinase activity was measured according to the absorbance at $340 \mathrm{~nm}$ owing to oxidation of NADH (7). The optical density was detected by Multimode Detector (DTX 880; Beckman Coulter). Kinetic assays for activity determinations contained cell lysate $(2 \mu \mathrm{g}), 1 \mathrm{~mol} / 1$ Tris- $\mathrm{HCl} \mathrm{pH} 8.0$ (0.05 ml), $1 \mathrm{~mol} / 1$ $\mathrm{KCl}(0.05 \mathrm{ml}), 0.1 \mathrm{~mol} / \mathrm{l} \mathrm{MgCl}_{2}(0.05 \mathrm{ml}), 30 \mathrm{mmol} / \mathrm{l} \mathrm{ADP}$ (0.025 ml), $2 \mathrm{mmol} / 1 \mathrm{NADH}(0.05 \mathrm{ml}), \mathrm{LDH}$ (12 units), $50 \mathrm{mmol} / 1 \mathrm{PEP}(0.05 \mathrm{ml})$ and adequate water to a total volume of $0.5 \mathrm{ml}$. The pyruvate kinase activity measured under the various CsA concentration indicated expression as the percentage of untreated to controls.

Statistical analysis. All experiments were repeated at least 3 times. Data are presented as the mean \pm SD. Data analysis was performed using SPSS for Windows version 16.0. Statistical significance was determined by Student's t-test, with values of $\mathrm{P}<0.05$ considered to be statistically significant.

\section{Results}

PKM2 is highly expressed in breast cancer cell lines. As reported previously, PKM2 was highly expressed in the 3 breast cancer cell lines MCF-7, MDA-MB-435 and MDA-MB231. The results showed that PKM2 mRNA was significantly overexpressed in breast cancer line MCF-7 as compared to the normal breast primary cells measured by qRT-PCR (Fig. 1A). 
A

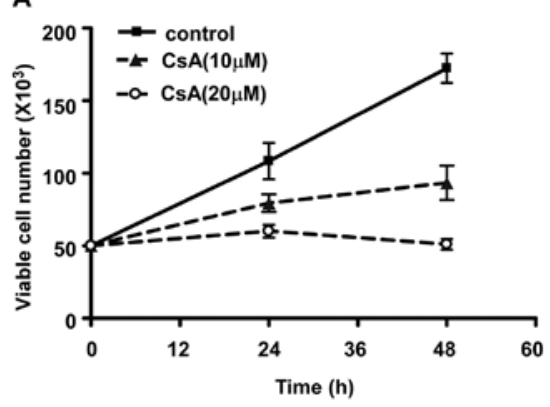

B
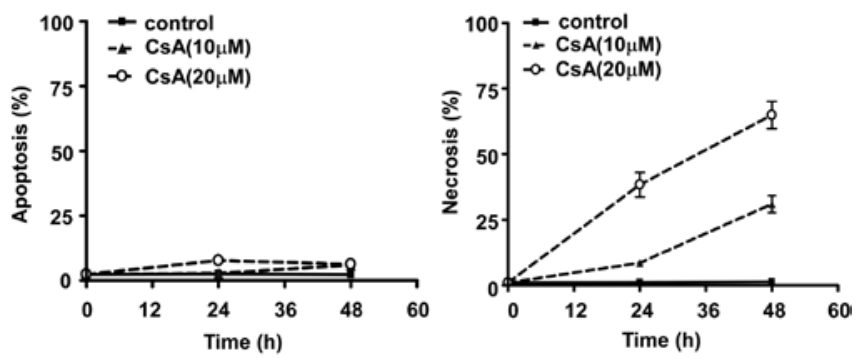

Figure 3. CsA treatment induced cell death of MCF-7 breast cancer cells. (A) Time- and dose-dependent inhibition of MCF-7 cells by CsA. MCF-7 cells were grown for 24 or $48 \mathrm{~h}$ in the presence of different concentrations of CsA as indicated. Viable cells were counted by trypan blue staining. Data are the mean $\pm S D(n=3)$ of a representative experiment. (B) The proportion of apoptotic $\left(\right.$ Annxin $\left.\mathrm{V}^{+}\right)$and necrotic $\left(\mathrm{PI}^{+}\right.$Annxin $\left.\mathrm{V}^{-}\right)$cells was determined by PI/Annxin V staining after treatment with 10 or $20 \mu \mathrm{M}$ CsA. Similar results were obtained in 3 independent experiments.

PKM2 mRNA was also overexpressed in MDA-MB-435 and MDA-MB-231 breast cancer cell lines (Fig. 1B). The protein level of PKM2 is upregulated in these breast cancer cell lines analyzed by western blotting (Fig. 1C). These results suggested that tumorigenesis is characterized by the transformation from tissue-specific PKM1 to tumor-specific PKM2.

Effect of CsA on cell growth and survival. Uncontrolled growth is an integral feature of all malignant tumors. In order to investigate the effect of CsA on breast cancer cell growth, MCF-7, MDA-MB-435, MDA-MB-231 and normal breast primary cells were cultured in the presence of various concentrations of CsA. The results showed that CsA significantly inhibited the growth of cancer cell lines in a dose-dependent manner, but the primary breast cells were much less sensitive to different concentrations of CsA (Fig. 2A). Flow cytometry analysis revealed that CsA induced cell cycle arrest in MCF-7 cells, as evidenced by the shift of cells from the S to the G1 phase (Fig. 2B).

Furthermore, treatment with CsA significantly decreased the number of viable MCF-7 cells in a time- and dosedependent manner (Fig. 3A). To determine whether CsA inhibited cell growth by inducing apoptosis of MCF-7 cells, we analyzed the percentage of apoptotic cells gated as positive Annexin-V staining (20) and necrotic cells gated as PI positive cell populations detected by the FACS assay. As shown in Fig. 3B, CsA induced MCF-7 cell death mainly via necrosis but not apoptosis and the cell death was time-dependent.

CsA inhibits the expression and the activity of PKM2 in breast cancer cells. In order to test the inhibitory effect of CsA on the pyruvate kinase $\mathrm{M}$ gene, qRT-PCR was performed to
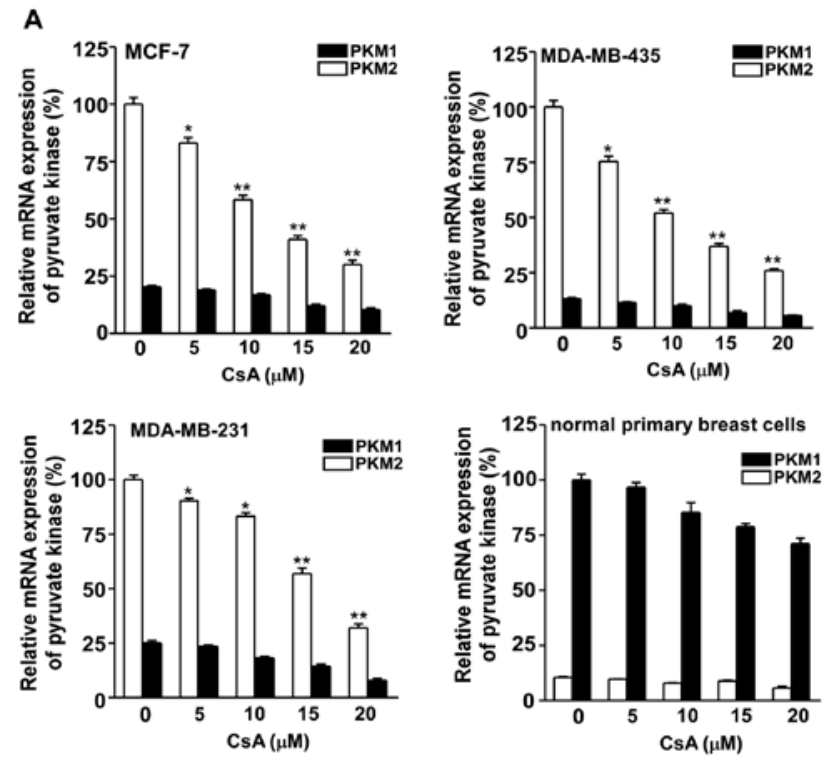

B

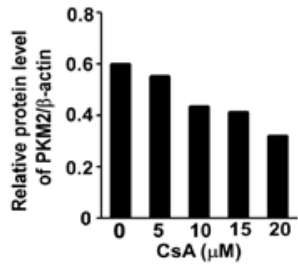

Figure 4. CsA downregulated the expression and the activity of PKM2 in breast cancer cells. (A) Expression of PKM1 and PKM2 in mRNA level was assayed by real-time PCR. MCF-7, MDA-MB-435, MDA-MB-231 and normal breast primary cells were cultured with various concentrations of CsA for $24 \mathrm{~h}$. Data are the mean $\pm \mathrm{SD}(\mathrm{n}=3)$ of a representative experiment. ${ }^{*} \mathrm{P}<0.05$; *** $\mathrm{P}<0.01$ vs. CsA-untreated cells. (B) The protein level of PKM2 was measured in MCF-7 cells after treated with various concentrations of CsA for $24 \mathrm{~h}$ by western blotting. The data shown are representative of 3 independent experiments.

measure the expression of PKM1/2 in MCF-7, MDA-MB435, MDA-MB-231 and breast primary cells. PKM2 mRNA expression was significantly inhibited in the 3 cancer cell lines following exposure to CsA (Fig. 4A). The protein level of PKM2 expression in MCF cells was also downregulated after the treatment with various concentrations of CsA (Fig. 4B).

Furthermore, the enzyme activity of PKM2, which catalyzes the dephosphorylation of phosphoenolpyruvate (PEP) to pyruvate was measured by reduction of NADH. As shown in Fig. 5A, CsA exposure caused a 5-40\% inhibition of PKM2 activity in MCF-7 cells compared to untreated cells with a dose-dependent manner. Pyruvate contributes to net adenosine triphosphate (ATP) production within the glycolytic pathway. The ATP production was detected in MCF cells after CsA exposure. It was showed that decrease of PKM2 activity was accompanied with reduction of ATP production (Fig. 5B). However, there was no difference in mitochondria function between untreated MCF-7 cells and cells treated with CsA (Fig. 5C).

All above data suggested that decreased PKM2 activity led to reduction of ATP production, which may result in cell death after CsA treatment in MCF-7 cells (21).

Inhibition of cell growth by CsA is mainly dependent on PKM2 activity. To determine whether the effect of CsA is PKM2 
A

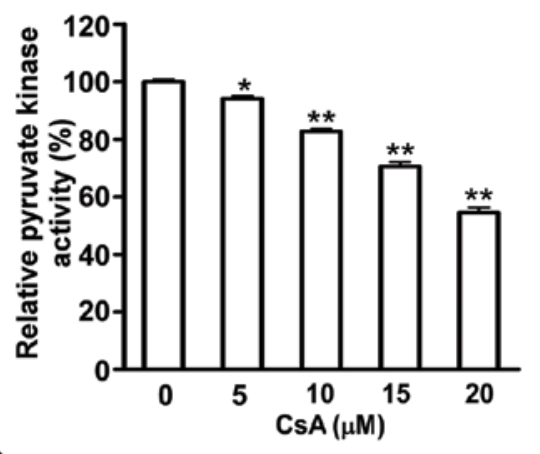

B

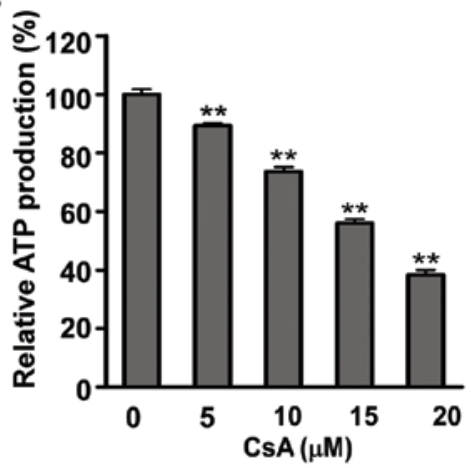

C
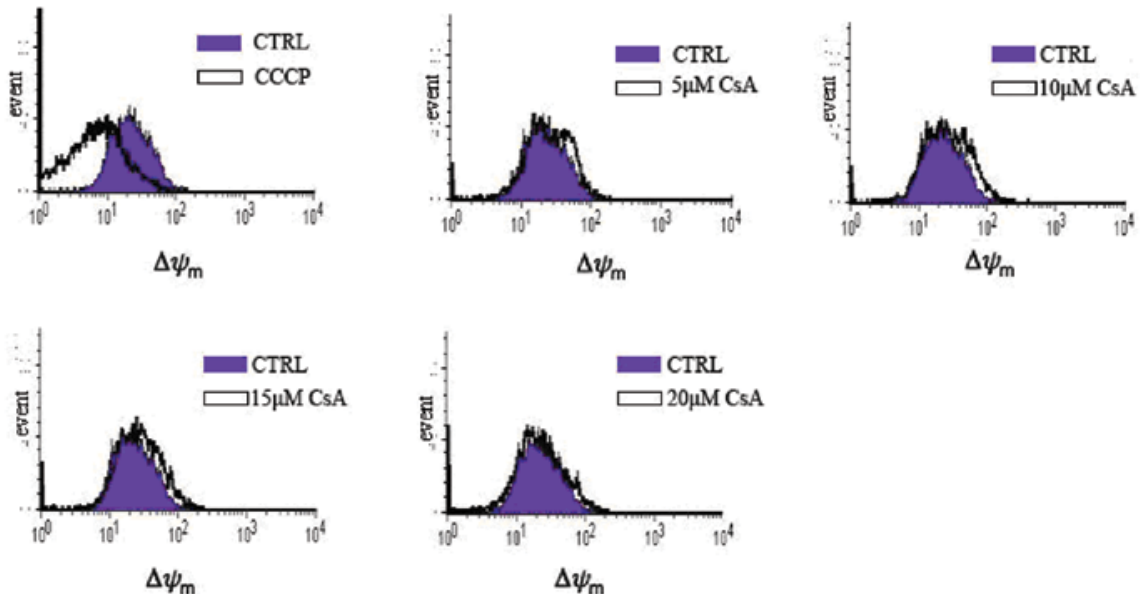

Figure 5. CsA inhibited the activity of PKM2 in MCF-7 cells. (A) Pyruvate kinase activity in MCF-7 cells after CsA exposure was detected according to the absorbance at $340 \mathrm{~nm}$ owing to oxidation of NADH. Data are the mean $\pm \mathrm{SD}(\mathrm{n}=4)$ of 1 representative experiment. ${ }^{*} \mathrm{P}<0.05$, ${ }^{* *} \mathrm{P}<0.01$ vs. CsA-untreated cells. (B) ATP concentration in MCF-7 cells after CsA treatment for $24 \mathrm{~h}$ was measured by the luminometric ATP assay. Data are the mean \pm SD ( $\mathrm{n}=3$ ) of a representative experiment. ${ }^{*} \mathrm{P}<0.05{ }^{* *} \mathrm{P}<0.01$ vs. CsA-untreated cells. (C) Mitochondrial transmembrane potential ( $\left.\Delta \Psi \mathrm{m}\right)$ in $\mathrm{MCF}-7$ cells was measured using JC-1 after treated with CCCP or various concentrations of CsA for $24 \mathrm{~h}$. The data shown are representative of 3 independent experiments.

dependent, we next investigated the role of ectopic overexpression of PKM2 to protect the MCF-7 cells from CsA-induced death. Interestingly, CsA failed to inhibit the proliferation of MCF-7 cells which were transiently transfected with PKM2pEGFP-N1 plasmid. As shown in Fig. 6A, a 3-5-fold increase in the expression of PKM2 was observed in MCF-7 cells after PKM2-pEGFP-N1 plasmid transfection, and CsA failed to downregulate the expression of PKM2 in MCF-7 cells with PKM2 plasmid transfection compared to the mock control (MCF-7 cells transfected with control pEGFP-N1 plasmid). Surprisingly, PKM2 expression was increased in transfected MCF-7 cells treated with CsA when compared to the transfected cells without CsA treatment. It may be due to the fact that the cells which did not gain the plasmid were inhibited by CsA, resulting in a high proportion of plasmid-gained cells.

The activity of PKM2 was also determined in plasmidtransfected cells after CsA exposure. It was showed that the activity of PKM2 was increased after transfection and that CsA failed to downregulate the enzyme activity (Fig. 6B). To further demonstrate that the ectopic overexpression of PKM2 induced resistance to CsA, MTT assay and cell cycle analysis were also used to determine the growth of MCF-7 cells. It was shown that ectopic overexpression of PKM2 protected MCF-7 cells from the inhibition effect of CsA (Fig. 6C) and partially reversed the cell cycle arrest induced by CsA (Fig. 6D).

\section{Discussion}

The metabolic state of tumor cells is different from that of normal counterparts and a high glycolytic capacity is crucial for cancer cell survival and proliferation (22). For this purpose, a switch of isoenzyme pattern and activity is initiated in metabolic phenotypes. Pyruvate kinase is a key enzyme of glycolysis. Different isoforms of this enzyme exist (pyruvate kinases L, R, M1, M2) and are tissue-specifically expressed in various organisms. PKM1 and PKM2 are different splicing products of the same gene (23). They differ only in 22 amino acid residues in an exon of 45 amino acids (24). The M1 isoform is expressed in most adult tissues while the M2 isoform is strongly overexpressed in most, if not all, cancers $(2,25)$. Recent studies have showed that, in human cancer cells, epidermal growth factor receptor (EGFR) activation induces translocation of PKM2, but not PKM1, into the nucleus where K433 of PKM2 binds to c-Src-phosphorylated Y333 of $\beta$-catenin. This interaction is required for both proteins to be recruited to the CCND1 promoter, leading to HDAC3 removal from the promoter, histone $\mathrm{H} 3$ acetylation and cyclin D1 expression. PKM2-dependent $\beta$-catenin transactivation is instrumental in EGFR-promoted tumor cell proliferation and brain tumor development (5). Many studies have indicated that PKM2 might be a promising target for cancer intervention 
A

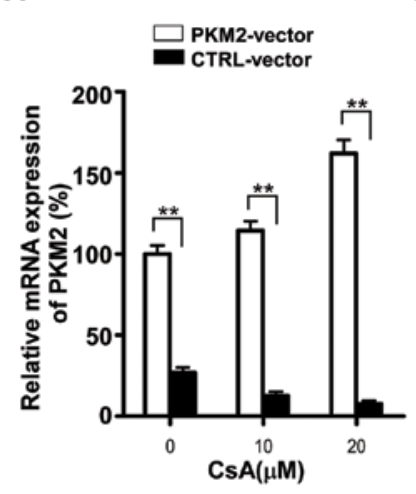

D

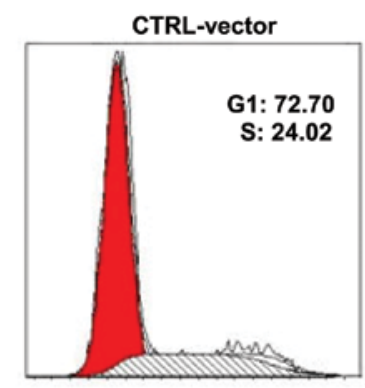

PKM2-vector

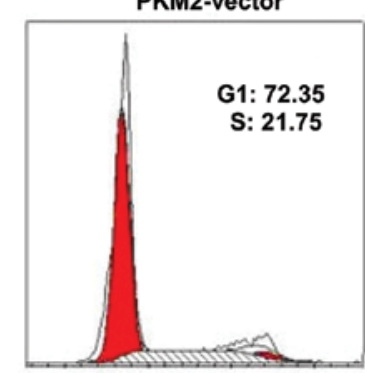

B

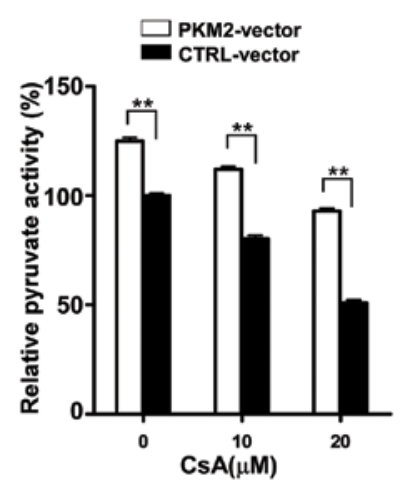

CTRL-vector+10 $\mu \mathrm{M}$ CsA

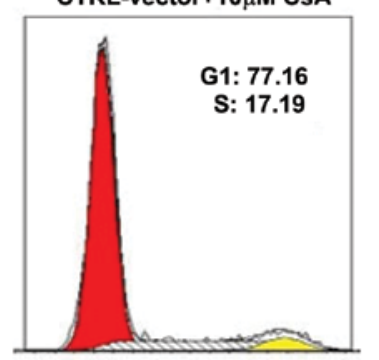

PKM2-vector+10 $\mu$ M CsA

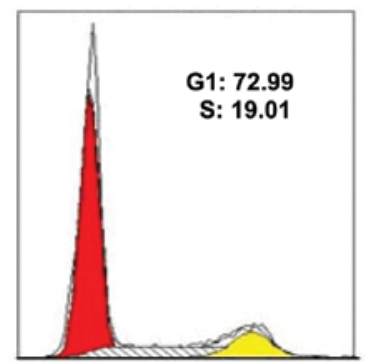

C
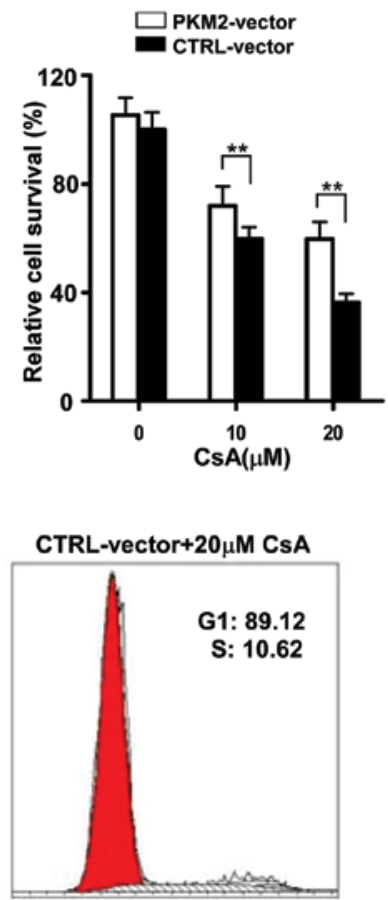

PKM2-vector $+20 \mu M$ CsA

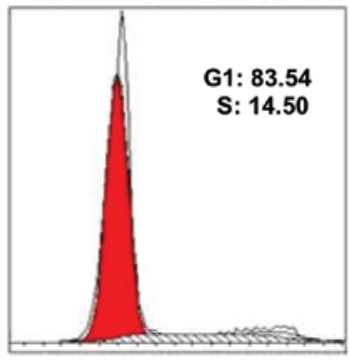

Figure 6. Inhibition of cell growth by CsA is mainly dependent on PKM2 activity. MCF-7 cells were transiently transfected with PKM2-pEGFP-N1 vector or control vector. (A) Relative mRNA expression of PKM2 in MCF-7 cells after transient transfection of PKM2 vector assayed by real-time PCR. Data are the mean $\pm \mathrm{SD}(\mathrm{n}=3)$ of one representative experiment. ${ }^{* *} \mathrm{P}<0.01$ vs. control vector transfected cells. (B) Relative pyruvate kinase activity in MCF-7 after transient transfection with PKM2-vector. Data are the mean $\pm \mathrm{SD}(\mathrm{n}=3)$ of a representative experiment. ${ }^{* *} \mathrm{P}<0.01$ vs. control vector transfected cells. (C) MTT assay and (D) cell cycle analysis showed that PKM2-vector transfection of MCF-7 cells partially inhibited the effect of CsA. Similar results were obtained in 3 independent experiments. ${ }^{* *} \mathrm{P}<0.01$ vs. control vector transfected cells.

$(3,4,25)$. Here we showed that the 3 breast cancer cell lines exhibited substantially high PKM2 expression as compared to the normal primary breast cells, suggesting that tumor M2-PK provided a good discrimination of benign disease from a malignant one, including breast cancers $(26,27)$.

We found that CsA suppressed the cell growth, cell cycle progression and G1/S phase transition of breast cancer cell lines. Recent studies showed that CsA inhibited the expression of some oncogenes. CsA could suppress cancer cell proliferation probably through regulating the expression levels of c-Myc, p21 and PCNA via inhibition of CaN/NFAT activity $(28,29)$. Our data demonstrated for the first time that CsA downregulated the cancer-specific PKM2 which promotes the progression of the tumor. The enzyme activity of PKM2 was also inhibited in MCF-7 cells treated with CsA. As pyruvate kinase catalyzes the last step in the glycolytic sequence and is responsible for net ATP production in this pathway, we measured the production of ATP in MCF-7 treated with CsA and found a significant reduction. This suggests that CsA caused inhibition of PKM2 expression, resulting in decrease of the intracellular ATP in tumor cells and then leading to a deceleration of cell proliferation and even induction of cell death. In order to confirm this view, we measured the function of mitochondria showing that the decrease in the ATP production is not dependent on mitochondria. Moreover, transient transfection with PKM2 expression vector into MCF-7 cells partially reversed the inhibition effect of CsA. Thus, we conclude that the most likely target of CsA action as inhibitor of breast cell growth is the tumor-specific PKM2.

As shown in our results, the sensitivity of the normal tissue cells was much lower than the tumor cell lines when treated with CsA. We supposed that the capacity of CsA to decrease the expression of PKM1 and PKM2 was different and M2-PK was more sensitive. There are some differences of function between M1-PK and M2-PK, a well known one is that the nonallosteric M1 isoenzyme exists only as a tetramer, which is a constitutively active enzyme while M2-PK is an allosteric enzyme that can exist in two different conformations (a tetrameric form which is 
active and a dimeric form which is inactive) $(17,30)$. A previous study has demonstrated that the tetramer:dimer ratio of M2-PK is directly controlled by different signal metabolites and oncoproteins (2). CsA decreased the expression and activity of pyruvate kinase M2 and thus reduced the production of ATP in cancer cells rather than that in normal tissues.

CsA is a kind of classic immunosuppressant, so general administration of CsA to patients with breast cancers may impair anti-tumor immune responses of the host and be detrimental. However, local administration of CsA may be beneficial because this could inhibit breast cancer proliferation without inhibiting general immunity. Moreover, this application might impair the suppressive function of $\mathrm{CD} 4^{+} \mathrm{CD} 25^{+} \mathrm{Foxp} 3^{+}$regulatory $\mathrm{T}$ cells (Tregs) which infiltrated into tumor tissues. Tregs are known to suppress anti-tumor immune responses $(31,32)$ and their differentiation and suppressive function are guided by the interaction of transcription factor Foxp3 with NFAT (33), which is also inhibited by CsA.

In conclusion, we demonstrated that CsA was able to effectively inhibit PKM2 overexpression in breast cancer cells, which is relative to cancer cell proliferation. Although it remains to be further investigated whether CsA treatment would be beneficial in breast cancer treatment, the mechanism suggested here might be useful to develop efficient approaches to treat breast cancers.

\section{Acknowledgements}

This study was supported by grants from the National Natural Science Foundation of China (81072405), the 'Program for New Century Excellent Talents in University' from the Ministry of Education of China (NCET08-0486) and the Zhejiang Provincial Natural Science Foundation of China (R2100528). It was also sponsored by the Zhejiang Provincial Program for the cultivation of high-level innovative health talents.

\section{References}

1. Warburg O: On the origin of cancer cells. Science 123: 309-314, 1956.

2. Mazurek S, Boschek CB, Hugo F, et al: Pyruvate kinase type M2 and its role in tumor growth and spreading. Semin Cancer Biol 15: 300-308, 2005.

3. Anastasiou D, Poulogiannis G, Asara JM, et al: Inhibition of pyruvate kinase M2 by reactive oxygen species contributes to cellular antioxidant responses. Science 334: 1278-1283, 2011.

4. Goldberg MS and Sharp PA: Pyruvate kinase M2-specific siRNA induces apoptosis and tumor regression. J Exp Med: Jan 23, 2012 (Epub ahead of print). doi: 10.1084/jem.20111487.

5. Yang W, Xia Y, Ji H, et al: Nuclear PKM2 regulates beta-catenin transactivation upon EGFR activation. Nature 480: 118-122, 2011.

6. Christofk HR, Vander Heiden MG, Harris MH, et al: The M2 splice isoform of pyruvate kinase is important for cancer metabolism and tumour growth. Nature 452: 230-233, 2008.

7. Christofk HR, Vander Heiden MG, Wu N, et al: Pyruvate kinase M2 is a phosphotyrosine-binding protein. Nature 452: 181-186, 2008.

8. Altenberg B and Greulich KO: Genes of glycolysis are ubiquitously overexpressed in 24 cancer classes. Genomics 84: 1014-1020, 2004.

9. Saeki T, Ueda K, Tanigawara Y, et al: Human P-glycoprotein transports cyclosporin A and FK506. J Biol Chem 268: 60776080, 1993.

10. Tan B, Piwnica-Worms D and Ratner L: Multidrug resistance transporters and modulation. Curr Opin Oncol 12: 450-458, 2000.
11. Englund G, Hallberg P, Artursson P, et al: Association between the number of coadministered P-glycoprotein inhibitors and serum digoxin levels in patients on therapeutic drug monitoring. BMC Med 2: 8, 2004.

12. Carcel-Trullols J, Torres-Molina F, Araico A, et al: Effect of cyclosporine A on the tissue distribution and pharmacokinetics of etoposide. Cancer Chemother Pharmacol 54: 153-160, 2004.

13. Xia CQ, Liu N, Miwa GT, et al: Interactions of cyclosporin A with breast cancer resistance protein. Drug Metab Dispos 35: 576-582, 2007.

14. Berthon P, Pancino G, de Cremoux P, et al: Characterization of normal breast epithelial cells in primary cultures: differentiation and growth factor receptors studies. In Vitro Cell Dev Biol 28A: 716-724, 1992.

15. Wang QQ, Li H, Oliver $\mathrm{T}$, et al: Integrin beta 1 regulates phagosome maturation in macrophages through Rac expression. J Immunol 180: 2419-2428, 2008.

16. Liu Y, Chen Q, Song Y, et al: MicroRNA-98 negatively regulates IL-10 production and endotoxin tolerance in macrophages after LPS stimulation. FEBS Lett 585: 1963-1968, 2011.

17. Dombrauckas JD, Santarsiero BD and Mesecar AD: Structural basis for tumor pyruvate kinase M2 allosteric regulation and catalysis. Biochemistry 44: 9417-9429, 2005.

18. Livak KJ and Schmittgen TD: Analysis of relative gene expression data using real-time quantitative PCR and the 2(-Delta Delta C(T)) method. Methods 25: 402-408, 2001.

19. Larson EL, Aiello AE, Gomez-Duarte C, et al: Bioluminescence ATP monitoring as a surrogate marker for microbial load on hands and surfaces in the home. Food Microbiol 20: 735-739, 2003.

20. Ishaque $A$ and Al-Rubeai $\mathrm{M}$ : Use of intracellular $\mathrm{pH}$ and annexin- $V$ flow cytometric assays to monitor apoptosis and its suppression by bcl-2 over-expression in hybridoma cell culture. J Immunol Methods 221: 43-57, 1998.

21. Leist M, Single B, Castoldi AF, et al: Intracellular adenosine triphosphate (ATP) concentration: a switch in the decision between apoptosis and necrosis. J Exp Med 185: 1481-1486, 1997.

22. Garber K: Energy deregulation: licensing tumors to grow. Science 312: 1158-1159, 2006.

23. Tani K, Yoshida MC, Satoh H, et al: Human M2-type pyruvate kinase: cDNA cloning, chromosomal assignment and expression in hepatoma. Gene 73: 509-516, 1988.

24. Yamada K and Noguchi T: Regulation of pyruvate kinase $M$ gene expression. Biochem Biophys Res Commun 256: 257-262, 1999.

25. Li R, Liu J, Xue H, et al: Diagnostic value of fecal tumor M2-pyruvate kinase for CRC screening: A systematic review and meta-analysis. Int J Cancer: Jan 19, 2012 (Epub ahead of print). doi: 10.1002/ijc.27442.

26. Schneider J, Velcovsky HG, Morr H, et al: Comparison of the tumor markers tumor M2-PK, CEA, CYFRA 21-1, NSE and SCC in the diagnosis of lung cancer. Anticancer Res 20: 5053-5058, 2000.

27. Schneider J and Schulze G: Comparison of tumor M2-pyruvate kinase (tumor M2-PK), carcinoembryonic antigen (CEA), carbohydrate antigens CA 19-9 and CA 72-4 in the diagnosis of gastrointestinal cancer. Anticancer Res 23: 5089-5093, 2003.

28. Buchholz M, Schatz A, Wagner M, et al: Overexpression of c-myc in pancreatic cancer caused by ectopic activation of NFATc1 and the $\mathrm{Ca} 2+/$ calcineurin signaling pathway. EMBO J 25: 3714-3724, 2006.

29. Masuo T, Okamura S, Zhang Y, et al: Cyclosporine A inhibits colorectal cancer proliferation probably by regulating expression levels of c-Myc, p21(WAF1/CIP1) and proliferating cell nuclear antigen. Cancer Lett 285: 66-72, 2009.

30. Wooll JO, Friesen RH, White MA, et al: Structural and functional linkages between subunit interfaces in mammalian pyruvate kinase. J Mol Biol 312: 525-540, 2001.

31. Gallimore A and Godkin A: Regulatory T cells and tumour immunity - observations in mice and men. Immunology 123: 157-163, 2008.

32. Curiel TJ, Coukos G, Zou L, et al: Specific recruitment of regulatory $\mathrm{T}$ cells in ovarian carcinoma fosters immune privilege and predicts reduced survival. Nat Med 10: 942-949, 2004.

33. Wu Y, Borde M, Heissmeyer V, et al: FOXP3 controls regulatory T cell function through cooperation with NFAT. Cell 126: 375-387, 2006. 